\title{
Adenoma hipofisiario en una paciente embarazada, evolución y manejo: Reporte de caso y revisión de la literatura
}

\author{
María Gimena Mejía López', Viviana Carolina Filizzola Montero ${ }^{2}$, Henry Tovar Cortés ${ }^{3}$, \\ William Rojas . $^{2}$.
}

\author{
${ }^{1}$ Médica, Residente de segundo año - Servicio de Endocrinología Hospital \\ San José \\ ${ }^{2}$ Médica, Residente de segundo año - Servicio de Endocrinología Hospital \\ San José \\ ${ }^{3}$ Médico Internista, Endocrinólogo - Instructor del programa de \\ Endocrinología Hospital San José \\ ${ }^{4}$ Médico Endocrinólogo - Instructor del programa de Endocrinología \\ Hospital San José
}

\section{Resumen}

S e reporta el caso de una paciente de 32 años quien consulta al servicio de endocrinología por crecimiento acral, hiperhidrosis, amenorrea, cefalea y cambios faciales. Se encuentra test de hormona de crecimiento postsupresión con $100 \mathrm{~g}$ de glucosa positivo e imagen de resonancia magnética nuclear con macroadenoma hipofisiario con quiasma libre. Se ordena resección de la masa e inicio de análogo de somatostatina; sin embargo, la paciente no inicia la medicación, además hay pérdida en el seguimiento clínico. Tiempo después consulta de nuevo al servicio de endocrinología en estado de embarazo. Se decide mantener en observación clínica durante la gestación, sin evidenciarse complicaciones secundarias a su patología ni en la madre ni en el neonato. Presentamos aquí la secuencia de hechos con sus resultados y una revisión de la literatura.

Palabras clave: Embarazo, adenoma hipofisiario, análogos de somatostatina.

\section{Abstract \\ This is the case of a 32-year-old female who was seen at the Endocrinology service complaining of acral growth, hyperhi- drosis, amenorrhea, headache and facial changes. The post-sup- pression growth hormone test with $100 \mathrm{~g}$ of glucose was positive and her MRI showed a pituitary macroadenoma without chiasm compression. Resection of the mass was scheduled followed by the use of a somatostatin analogue. However, the patient did not initiate the medication, and was lost for follow-up. Later on she visited again the Endocrinology Service while pregnant. It}

was decided to keep her under clinical observation during her pregnancy, with no evidence of complications secondary to the condition neither for the mother nor for the newborn. We report here the sequence of events with their results and a review of the literature.

Key words: Pregnancy, pituitary adenoma, somatostatin analogues.

\section{Presentación del caso}

Paciente de sexo femenino, de 32 años de edad, ama de casa, sin antecedentes médicos de importancia, que consulta al servicio de endocrinología en septiembre de 2013 por crecimiento acral, hiperhidrosis, cefalea global, cambios en los rasgos faciales, artralgias y amenorrea de dos años de evolución. Como antecedentes ginecobstétricos: menarquía a los 13 años con ciclos regulares hasta hace un año. G1P1A0, parto hace 3 años y FUM en julio 2 de 2013.

Al examen físico signos vitales normales. Peso: $65 \mathrm{Kg}$, Talla $1.53 \mathrm{~m}$, IMC: $27.8 \mathrm{Kg} / \mathrm{m}^{2}$. Se encuentra prognatismo, aumento de tejidos blandos en nariz y pabellones auriculares, diastema, macroglosia y acrocrecimiento en manos y pies.

Se ordena somatomedina C: $1.140 \mathrm{ng} / \mathrm{mL}$, realización de test de GH postsupresión de $100 \mathrm{~g}$ de glucosa, con los siguientes resultados: Basal: $21 \mathrm{ng} / \mathrm{mL}, 30 \mathrm{~min}: 31 \mathrm{ng} / \mathrm{mL}, 60 \mathrm{~min}: 56$ ng/ml, 90 min: 83 ng/mL y 120 min: 91 ng/mL. Prueba de embarazo negativa, prolactina $60 \mathrm{ng} / \mathrm{mL}$. Con estos resultados se ordena la toma de una resonancia magnética nuclear de silla turca (RMN) y un perfil hipofisiario basal (tabla 1).

Asiste nuevamente a consulta en noviembre de 2013 con RMN de silla turca que muestra un macroadenoma hipofisiario de 2,28 x 2,23 x 1,68 cm, sin compromiso del quiasma (figura 1).

Con estos elementos se realiza el diagnóstico de acromegalia secundaria a macroadenoma productor de $\mathrm{GH}$, se propone manejo quirúrgico. Es remitida para valoración por neurocirugía y se formula terapia con octréotide lar $20 \mathrm{mg}$ IM mensual en diciembre de 2013; sin embargo, la paciente no regresa a consulta por el servicio de endocrinología hasta marzo de 


\begin{tabular}{c|c}
\hline \multicolumn{2}{c}{ Tabla 1. Reportes de paraclínicos de la paciente } \\
Laboratorio & Inicial \\
\hline Cortisol & $13 \mathrm{mcg} / \mathrm{dL}$ \\
\hline Glicemia & $88 \mathrm{mg} / \mathrm{dL}$ \\
\hline Prolactina & $60 \mathrm{ng} / \mathrm{mL}$ \\
\hline Sodio & $132 \mathrm{mmol} / \mathrm{L}$ \\
\hline TSH & $1,50 \mathrm{mUl} / \mathrm{L}$ \\
\hline T4 libre & $1,03 \mathrm{mUl} / \mathrm{L}$ \\
\hline
\end{tabular}

2014, manifestando que no le autorizaron la medicación y adicionalmente con reciente prueba de embarazo positiva y ecografía pélvica que reporta embarazo de 19 semanas. Por lo cual se cambia la conducta de manejo temporalmente, considerando continuación de seguimiento clínico estricto hasta evidencia de nuevos cambios que requieran tratamiento o en caso contrario hasta finalizar la gestación.

Presenta parto vaginal eutócico en julio de 2014 con controles prenatales normales. Recién nacido con peso: 3.000 g y talla $48 \mathrm{~cm}$. Se realiza una campimetría visual que es normal y se da orden nuevamente para valoración por neurocirugía.

\section{Discusión}

Los tumores hipofisarios representan de $10 \%$ a $25 \%$ de todas las neoplasias intracraneales, y se pueden clasificar en tres grupos, de acuerdo con su comportamiento biológico ${ }^{(1)}$ :

- Adenomas benignos

- Adenomas invasivos

- Carcinoma

Los adenomas comprenden la mayor parte de los tumores hipofisiarios, con una incidencia general estimada de aproximadamente $17 \%$, pero sólo una minoría es sintomática ${ }^{(2)}$. Asimismo, los adenomas hipofisiarios se dividen desde el punto de vista anatómico en: intrahipofisarios intraselares, difusos e invasivos $^{(3)}$. Los adenomas invasivos que constituyen aproxi-

Figura 1. RMN de silla turca con contraste. a) Corte sagital en T1. b) Corte coronal en T1

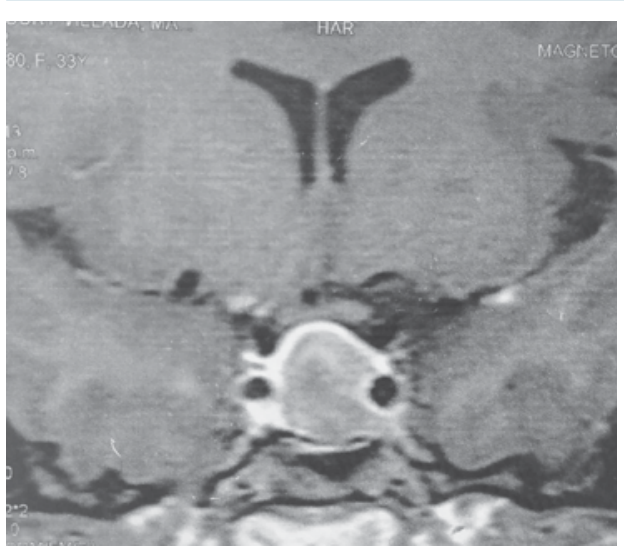

madamente $35 \%$ pueden invadir la duramadre, el hueso del cráneo o el seno esfenoidal ${ }^{(4)}$. Los carcinomas constituyen 0,1 a $0,2 \%$ de todos los tumores hipofisiarios ${ }^{(5,6)}$.

Durante la gestación se hace más difícil la interpretación de las imágenes hipofisiarias así como de las pruebas de laboratorio, tanto basales como posestímulo, en el diagnóstico y seguimiento de la patología hipofisiaria. Con base en el caso clínico descrito, discutiremos aquellos adenomas pituitarios que se presentan con mayor prevalencia durante la gestación ${ }^{(7)}$.

Durante el embarazo, fisiológicamente se presenta un aumento del tamaño de la pituitaria, debido al incremento de su flujo sanguíneo y a la hiperplasia de las células lactotropas, encontrándose una hipófisis convexa y agrandada; sin embargo, el crecimiento asimétrico, la altura mayor a $10 \mathrm{~mm}$ y la desviación del tallo hacen pensar en un proceso patológico ${ }^{(7)}$.

En el caso de los prolactinomas en pacientes no embarazadas u hombres, la búsqueda por imágenes se inicia con valores de prolactina no inferiores a $100 \mathrm{ng} / \mathrm{mL}$, así como es infrecuente encontrar valores menores en incidentalomas productores de prolactina. En el embarazo, debido al gran incremento de estrógenos y progesterona, y a la producción de prolactina por la decidua placentaria de forma independiente a la supresión de dopamina y TRH, los niveles de prolactina se encuentran en promedio 10 veces por encima de lo esperado en no embarazadas, siendo normales valores de $200 \mathrm{ng} / \mathrm{mL}$ o mayores, lo cual dificulta tanto el estudio bioquímico inicial como el seguimiento; teniendo en cuenta que los prolactinomas ocupan el 40\% de tumores hipofisiarios en la gestación ${ }^{(8,9)}$.

Tanto en pacientes no embarazadas y hombres, como en las gestantes, la primera línea de tratamiento es el uso de agonistas dopaminérgicos ${ }^{(10)}$.

La recomendación de manejo en aquellas pacientes con diagnóstico de tumor productor de prolactina con deseos de gestación depende del tamaño tumoral, ya que la complicación temida en el embarazo es la compresión de estructuras vecinas por crecimiento del tumor. De esta forma, con un riesgo de crecimiento clínicamente significativo de 2,6\% para microprolactinomas durante el embarazo, la recomendación es continuar el manejo médico con agonista dopaminérgico hasta lograr la concepción y suspenderlo en cuanto se confirme la gestación. Por el contrario, el riesgo en macroprolactinomas asciende a $31 \%$, por lo cual la indicación es la resección quirúrgica antes de intentar el embarazo, reduciendo de esta manera la probabilidad de compresión con las implicaciones clínicas por crecimiento al $5 \%{ }^{(11)}$. 
Si el diagnóstico se realiza durante el embarazo la conducta depende del compromiso clínico por compresión. De esta forma, tanto en los tumores diagnosticados antes del embarazo como durante la gestación, el seguimiento del prolactinoma es netamente clínico, vigilando constantemente la aparición de cefalea severa, aumento en la intensidad de la misma o empeoramiento de la capacidad visual. En estos casos es mandatoria la realización de resonancia magnética nuclear (RMN) y campimetría visual. En ninguna otra situación se recomienda de rutina la realización de estudios bioquímicos ni imaginológicos ${ }^{(9,11)}$.

Aunque los estudios no han demostrado relación directa entre uso de agonistas dopaminérgicos y efectos teratogénicos en el feto o complicaciones durante el embarazo, la guía recomienda suspender su uso una vez se confirme la gestación; sin embargo, en caso de crecimiento sintomático del tumor, se indica el inicio de bromocriptina como primera elección ${ }^{(12-15)}$.

En el caso específico de acromegalia, la situación no es muy diferente. Durante el primer trimestre, la hormona de crecimiento circulante es de origen hipofisiario y secretada en forma pulsátil, mientras que en el segundo y tercer trimestre predomina la GH de origen placentario (GHp), la cual es secretada en forma continua y se une con igual afinidad que la hipofisiaria al receptor de GH hepático, estimulando por sí misma la síntesis y secreción de IGF-1, induciendo retroalimentación negativa sobre las células somatotropas y reduciendo de esta forma, los niveles de somatotropina hipofisiaria ${ }^{(16,17)}$.

Los niveles séricos de $\mathrm{GH}$, pese a lo anterior, se encuentran elevados, debido a que distinguir entre la producción de GH hipofisiaria vs. placentaria durante la gestación es una tarea que los estudios de radioinmunoanálisis convencional (RIA), no pueden realizar. La medición de IGF-1 sérico tampoco es de utilidad, dado que la presencia de valores fisiológicamente elevados en el embarazo y patológicos por adenoma somatotropo pueden ser los mismos. La respuesta a la prueba de supresión con carga de glucosa tampoco brinda información adicional por que ni la placenta ni el adenoma productor de GH responden a la carga de glucosa con reducción de la secreción de $\mathrm{GH}^{(7,17,18)}$.

Teniendo en cuenta que en aquellas pacientes con adenoma hipofisiario existe una liberación autónoma de GH independiente de los niveles de IGF-1 de forma similar a la secreción de GHp, no se encontrará diferencia en el ritmo de pulsatilidad de GH que permita diferenciar picos de secreción de adenoma vs fisiológicos del segundo trimestre. Sin embargo, esta es una opción viable durante el primer trimestre del embarazo, cuando prima la síntesis de GH hipofisiaria sobre la $\mathrm{GHp}^{(7,16)}$.

A pesar de la poca confiabilidad de los estudios bioquímicos, que no nos permiten un diagnóstico paraclínico durante el embarazo, en caso de alta sospecha clínica de acromegalia durante el curso de la gestación, está justificado el uso de RMN sin gadolinio, sin olvidar que debido a los cambios fisiológicos de la glándula hipófisis es posible observar falsos positivos y que la ausencia de contraste predispone en la lectura a falsos negativos en tumores pequeños. Sin embargo, la utilidad de este estudio radica en la evaluación del compromiso de estructuras vecinas, lo que lo hace útil en el seguimiento de pacientes con diagnóstico pregestacional de acromegalia con síntomas de novo o en progresión de compromiso del campo visual, confirmado previamente con campimetría computarizada ${ }^{(7,13,16,18)}$.

Los pocos estudios realizados hasta la fecha muestran baja prevalencia de crecimiento del tumor durante el embarazo, siendo menor en microadenomas y en tumores previamente operados o irradiados ${ }^{(7,18,19)}$, las complicaciones asociadas a la hipersecreción de GH incluyen intolerancia a los carbohidratos en el $60 \%$, diabetes mellitus en $13-32 \%$, otras como cefalea, hipertensión arterial y con menor frecuencia preeclampsia, pero en general las pacientes completan el embarazo asintomáticas, incluso se describe mejoría de los síntomas previos a la concepción; los eventos adversos sobre teratogenicidad fetal y complicaciones perinatales no representan ninguna asociación clara con la enfermedad(7,18-21).

Existe escasa evidencia sobre la seguridad y efectividad del tratamiento farmacológico en gestantes con acromegalia. Los agonistas dopaminérgicos no han demostrado, como se describió anteriormente, estar asociados a desenlaces adversos fetales. Estos medicamentos cruzan la barrera placentaria, pero hay evidencia que soporta la hipótesis de un mecanismo de protección fetal a nivel placentario que evita efectos secundarios fetales, como lo muestran los pocos estudios disponibles, con algunos casos de baja talla o peso al nacer, pero con predominio de embarazos exitosos y recién nacidos sanos. El uso de pegvisomant ha sido poco estudiado; sin embargo, hasta el momento la evidencia no ha demostrado efectos adversos materno-fetales secundarios en casos reportados de toma desde antes del inicio del embarazo o de inicio en etapas tempranas de la gestación y no se excreta por leche materna. Debido a la falta de evidencia, se recomienda la suspensión de los mencionados medicamentos dos meses antes de un embarazo planeado o al conocer el diagnóstico de embarazo, sólo se permite su uso en casos de empeoramiento de la clínica de acromegalia y reexpansión del adenoma ${ }^{(16,18-25)}$. La cirugía sólo está indicada en caso de crecimiento tumoral con síntomas compresivos y síntomas visuales de novo o en progresión ${ }^{(16,18,21)}$.

La prevalencia de la enfermedad de Cushing en el embarazo es menor debido a la baja probabilidad de embarazo en estas pacientes.

Durante la gestación, se genera hipercortisolismo fisiológico y adaptativo debido a la síntesis placentaria de CRH con actividad sobre las células corticotropas hipofisiarias, aumento subsiguiente del cortisol libre adrenal e incremento del cortisol total por acción de los estrógenos placentarios sobre los niveles de proteína unidora de cortisol. A pesar de esta hipercortisolemia, la elevada concentración de progesterona ejerce un efecto regulador que impide la presentación de síntomas de Cushing en la madre y se mantiene el ritmo circadiano nor- 
mal del cortisol. Por su parte la intensa actividad de la isoenzima $11 \beta$ - HSD placentaria inactiva el cortisol evitando efectos de hipercortisolismo en el feto ${ }^{(16,26)}$.

La enfermedad de Cushing es responsable de $30 \%$ a $35 \%$ de los casos de síndrome de Cushing en el embarazo. Los síntomas son similares a la población general, sin embargo, suelen confundirse con los cambios normales del embarazo, lo cual hace más difícil el diagnóstico ${ }^{(7,27)}$.

Los valores normales de cortisol 11 pm se mantienen un poco elevados en el embarazo respecto a la población general, en niveles menores de 7,5 mg/dL o $50 \%$ de los niveles de la mañana, dado que la variación circadiana del cortisol se mantiene durante el embarazo, la pérdida de ésta sugiere producción autónoma. El cortisol libre en orina de 24 horas parece ser la mejor opción como prueba inicial en el estudio de Cushing en gestantes, tomando en el primer trimestre valores por encima de $200 \mathrm{mg} / 24 \mathrm{~h}$, y mayor de tres veces el límite superior en los dos últimos trimestres. El test de dexametasona se desaconseja por alta tasa de falsos $\operatorname{positivos}^{(16,28,29)}$.

Las principales complicaciones maternas son: hipertensión arterial severa $67 \%$, diabetes gestacional 30\% e insuficiencia cardiaca, alcanzando una mortalidad del 4\%. En 33-
$60 \%$ de los fetos se presenta prematuridad, en $26 \%$ retraso de crecimiento intrauterino, en $26 \%$ aborto espontáneo y en $17 \%$ muerte fetal ${ }^{(7,16,27,28)}$

El tratamiento de primera línea es la resección transesfenoidal del adenoma en la semana 12 a 29 de gestación, el manejo médico sólo se indica en caso de síntomas severos e imposibilidad de manejo quirúrgico. Los medicamentos más estudiados durante el embarazo son ketoconazol y metirapona que ocupan la categoría $\mathrm{C}$ de teratogenicidad por la FDA, con descripción de parto pretérmino y restricción del crecimiento intrauterino como los efectos adversos más frecuentes; a pesar de esto, el tratamiento se ha asociado con mejoría en los resultados maternos y fetales, comparados con las mujeres no tratadas ${ }^{(16,29,30)}$.

Si bien los adenomas hipofisiarios funcionantes más frecuentes son los tres presentados en esta revisión, se debe recordar la baja probabilidad de embarazo en mujeres con prolactinoma, acromegalia y síndrome de Cushing, en todos los casos, debido al hipogonadismo subsecuente. Finalmente, en pacientes con Cushing, el hipogonadismo es más marcado, ya que adicional al estado de hipercortisolismo las pacientes presentan hiperandrogenismo.

\section{Referencias}

1. Asa SL, Ezzat S: The cytogenesis and pathogenesis of pituitary adenomas. Endocr Rev 19 (6): 798-827, 1998.

2. Ezzat S, Asa SL, Couldwell WT, et al.: The prevalence of pituitary adenomas: a systematic review. Cancer 101 (3): 613-9, 2004.

3. Kovacs K, Horvath E, Vidal S: Classification of pituitary adenomas. J Neurooncol 54 (2): 121-7, 2001.

4. Scheithauer BW, Kovacs KT, Laws ER Jr, et al.: Pathology of invasive pituitary tumors with special reference to functional classification. J Neurosurg 65 (6): 733-44, 1986.

5. Pernicone PJ, Scheithauer BW, Sebo TJ, et al.: Pituitary carcinoma: a clinicopathologic study of 15 cases. Cancer 79 (4): 804-12, 1997.

6. Ragel BT, Couldwell WT: Pituitary carcinoma: a review of the literature. Neurosurg Focus 16 (4): E7, 2004.

7. Karaca Zuleyha, MD, Fahrettin Kelestimur. Pregnancy and other pituitary disorders(including GH deficiency) Best Practice \& Research Clinical Endocrinology \& Metabolism 25 (2011) 897-910.

8. Karaca, Z. F Tanriverdi, K Unluhizarci and F Kelestimur. Pregnancy and pituitary disorders. European Journal of Endocrinology (2010) 162 453-475.

9. Motivala et al. Endocrinol Metab Clin N Am 40 (2011) 827-836.

10. Maiter, Dominique, Primeau, Vanessa. 2012 Update in the treatment of prolactinomas. Annales d'Endocrinologie 73 (2012) 90-98.

11. Melded S, Casanueva FF, Hoffman AR, Kleinberg DL, Montori VM, Schlechte JA, Wass JA; Endocrine Society. Diagnosis and treatment of hyperprolactinemia: an Endocrine Society clinical practice guideline. J Clin Endocrinol Metab. 2011 Feb;96(2):273-88

12. Gillam MP, Molitch ME, Lombardi G, Colao A. Advances in the treatment of prolactinomas. E ndocr Rev. 27:485-534, 2006.

13. Molitch M.E. Prolactinoma in pregnancy Best Practice \& Research Clinical Endocrinology \& Metabolism 25 (2011) 885-896.

14. Konopka P, Raymond JP, Merceron RE, Seneze J. Continuous administration of bromocriptine in the prevention of neurological complications in pregnant women with prolactinomas. Am J Obstet Gynecol 146:935-938. 1983.

15. Jai Bhagwan Sharma, Kallol K. Roy, Pushparaj Mohanraj, Sunesh Kumar, Debjyoti Karmakar, Jinee Barua.

16. Bronstein, M. D. et al. Management of pituitary tumors in pregnancy. Nat. Rev. Endocrinol. 7, 301-310 (2011).
17. Cheng V., et al: Pregnancy and acromegaly: a review. Pituitary. 2012 Mar; 15(1):59-63.

18. Katznelson, Laurence, L, John. D. Atkinson. Cook, David M. Shereen Z. Ezzat, Amir H. Hamrahian,. Miller Karen K. American Association of Clinical Endocrinologists Medical Guidelines for Clinical Practice. Endocrine Practice Vol.17 (suppl 4) Jul/ago 2011.

19. Dias Monike, Boguszewski,Cesar. Gadelha, Monica. Kasuzi, Leandro. Musolino,Nina. Vieira, J. and Abucham ,J. Acromegaly and pregnancy: a prospective study. European Journal of Endocrinology.

20. Caron $\mathrm{Ph}$, et al: Acromegaly and pregnancy: a retrospective multicenter study of 59 pregnancies in 46 women. A JCEM 2010; 95:4680-87.

21. Cheng Sonia, Grasso Ludovica, Jose A. Martinez-Orozco, Rany Al-Agha, Pivonello Rosario, Colao Ana Maria and Ezzat Shereen. Pregnancy in acromegaly: experience from two referral centers and systematic review of the literatura. Clinical Endocrinology (2012) 76, 264-271.

22. Fleseriu M, m.d, et al: Acromegaly: a review of current medical therapy and new drugs on the horizon. Neurosurg Focus 29 (4):E15, 2010.

23. Riddle Brian, Susan. Bidlingmaier,Martin.P, Michael . Wajnrajch, Stuart A. Weinzimer, and Inzucchi, Silvio. Treatment of Acromegaly with Pegvisomant during Pregnancy: Maternal and Fetal Effects. The Journal of Clinical Endocrinology \& Metabolism . (2007)92(9):3374-3377.

24. Valassi Elena. Acromegaly and pregnancy. Endocrinol Nutr. 2013;60(1):1-3.

25. Van der Lely AJ1, Gomez R, Heissler JF, Åkerblad AC, Jönsson P, CamachoHübner C, Kołtowska-Häggström M. Pregnancy in acromegaly patients treated with pegvisomant. Endocrine. 2014 Dec 27.

26. Bronstein $\mathrm{M}$, et al: Medical management of pituitary adenomas; the special case of management of the pregnant woman. Pituitary. 2002;5:99-107.

27. Dreyfus M, Lehmann M, Schlienger JL. Patología endocrina y embarazo Enciclopedia médicoquirúrgica Madrid: Elsevier. 2003; tomo 4:5048-C-10.

28. López $\mathrm{S}$ et al. Síndrome de Cushing en el embarazo. Prog Obstet Ginecol. 2006;49(4):205-9.

29. Nieman, Lynnette K. M, Beverly . Biller. Newell-Price, Jonh. Savage, Martin. Stewart, Paul. And. Montori, Victor M. The medical management of Cushing's syndrome during pregnancy. European Journal of Obstetrics \& Gynecology and Reproductive Biology 168 (2013) 1-6.

30. Wei How Lim, David J. Torpy, William S. Jeffries. The medical management of Cushing's syndrome during pregnancy. European Journal of Obstetrics \& Gynecology and Reproductive Biology 168 (2013) 1-6. 